\title{
Original
}

\section{Light-induced Membrane Hyperpolarization Promotes Osteoblast Differentiation in MC3T3 Osteoblast-like Cells}

\author{
Takuya Notomi $^{1-3)}$, Ryuichiro Kobayashi ${ }^{2)}$, Miki Otsuka ${ }^{2)}$, Chie Kise ${ }^{2)}$, Yoshihiro Momota ${ }^{4)}$, Yoichi Ezura ${ }^{3)}$ and Takayoshi Kawazoe ${ }^{5)}$ \\ 1) Institute of Dental Research, Osaka Dental University, Osaka, Japan \\ 2) Department of Pharmacology, Osaka Dental University, Osaka, Japan \\ 3) Department of Molecular Pharmacology, Medical Research Institute, Tokyo Medical and Dental University, Tokyo, Japan \\ 4) Department of Anesthesiology, Osaka Dental University, Osaka, Japan \\ 5) Council, Osaka Dental University, Osaka, Japan \\ (Accepted for publication, September 6, 2021)
}

\begin{abstract}
Bone mass is regulated by bone remodeling, which involves bone formation by osteoblasts and bone resorption by osteoclasts. To prevent and treat bone loss, a basic understanding of the mechanism of bone formation is essential, including osteoblast differentiation, and its responses to mechanical stimuli that induce changes in membrane potential. During osteoblast differentiation, hyperpolarized membrane potential was observed. To understand osteoblast differentiation in response to membrane hyperpolarization, as well as the long-term effects of changes in membrane potential, we developed a light-controllable membrane potential system in MC3T3-E1 osteoblast-like cells by stably expressing the light-driven outward proton pump, archaerhodopsin-3. Archaerhodopsin-3 activation by yellow-green light hyperpolarizes the cell membrane Light-induced hyperpolarization accelerated osteoblast mineralization, as assessed by Alizarin Red staining, alkaline phosphatase activity, and expression levels of osteoblast differentiation markers. This promotion of osteoblast mineralization is related to voltage-gated $\mathrm{Ca}^{2+}$ channels. Our study revealed a novel role of membrane potential in non-excitable osteoblast-like cells.
\end{abstract}

Key words: Osteoblast, Opto-genetics, Hyperpolarization, Membrane potential

\section{Introduction}

Bone mass is maintained by bone-forming osteoblasts and bone-resorbing osteoclasts. An imbalance between osteoblasts and osteoclasts causes bone loss and subsequent osteoporosis ${ }^{1,2)}$. The mechanism of osteoblast differentiation has been well investigated to treat bone loss by increasing bone formation. These studies have highlighted the importance of transcription factors and growth factors, including bone morphogenetic proteins and insulin-like growth factors, and also implicate the involvement of calcium influx and voltage-gated ion channels ${ }^{3-6}$. However, the relationship between changes in membrane potential and osteoblast differentiation remains unclear, possibly because of the difficulty in controlling the membrane potential non-invasively.

Over the decades, studies on optogenetics have been reported mainly in excitable cells, such as neuronal cells. In channel rhodopsin- or archaerhodopsin-3 (Arch)-expressing neuronal cells and tissues, the light stimulus enables depolarization and/or hyperpolarization of the cell membrane and regulates the action potential ${ }^{7-9)}$. It remains challenging to examine the relationship between experimental and physiological changes in membrane potential in non-excitable cells. However, in osteoblasts, we demonstrated the physiological significance of membrane potential depolarization, the condition in which the membrane potential becomes positive ${ }^{10)}$. To regulate the membrane potential non-invasively

Correspondence to: Dr. Takuya Notomi, Institute of Dental Research, Osaka Dental University, 8-1 Kuzuhahanazono-cho, Hirakata-shi, Osaka 573-1121, Japan; Tel: +81-72-864-3096; Fax: +81-72-864-3096; E-mail: notomi@cc. osaka-dent.ac.jp in osteoblasts, we developed a membrane potential-controllable system using osteoblasts based on the light-activated protein rhodopsin. Transient membrane depolarization promoted the transport of receptor activator of nuclear factor kappa-B ligand (RANKL) lysosomal vesicles to the cell membrane within a few seconds, while the role of membrane hyperpolarization (the negative membrane potential) was not investigated. The long-term effects of changes in the membrane potential of osteoblasts are unknown.

Here, we show that the membrane potential is hyperpolarized during osteoblast differentiation and that light-induced membrane hyperpolarization promotes osteoblast differentiation in osteoblast-like cells that stably express the light-driven outward proton pump, $\mathrm{Arch}^{7}$. A once daily light stimulus throughout the mineralization period was sufficient to increase the expression levels of osteoblast differentiation markers and promote mineralization, as assessed by Alizarin Red staining and alkaline phosphatase (ALP) activity. This promotion of osteoblast mineralization by light-induced hyperpolarization was related to the contribution of voltage-gated $\mathrm{Ca}^{2+}$ channel (Cav). These results indicate that membrane potential plays a key role in cell differentiation in non-excitable osteoblasts.

\section{Materials and Methods \\ Cell culture and osteogenic assay \\ MC3T3-E1 clone4 osteoblast-like cells (OBs; American Type Cul- ture Collection, Manassas, VA, USA) were cultured in $\alpha$-MEM (Thermo Fisher Scientific K.K., Tokyo, Japan) supplemented with 10\% fetal bo-}


vine serum and $1 \%$ penicillin and streptomycin. All cells were maintained at $37^{\circ} \mathrm{C}$ with $5 \% \mathrm{CO}_{2}$ in a humidified atmosphere. All chemicals used were purchased from Wako Pure Chemical Industries (Osaka, Japan) unless otherwise specified. For osteogenic induction, OBs were treated with $100 \mu \mathrm{g} / \mathrm{ml}$ ascorbic acid, $10 \mathrm{mM} \beta$-glycerophosphate, and 1 $\mu \mathrm{M}$ dexamethasone in $\alpha-\mathrm{MEM}^{5)}$. To quantify the early stage of osteoblast differentiation, ALP activity and calcium mineralization were determined and evaluated according to methods described in previous studies $^{11,12}$. To view the mineralization process, cells were first fixed in a $3.7 \%$ formaldehyde solution, rinsed with deionized $\mathrm{H}_{2} \mathrm{O}$, and stained with Alizarin Red S for $20 \mathrm{~min}$ at $22 \pm 3^{\circ} \mathrm{C}$, after which excess dye was removed. Fluorescence or bright-field images were obtained using microscopy and subjected to quantitative analysis software (All-in-One Microscopy; Keyence, Osaka, Japan). The stained areas in each well were measured and normalized to the entire well area. To quantify the early stage of osteoblast differentiation at day 12 after the induction of osteoblast mineralization, ALP activity was measured using LabAssay ALP.

\section{Establishment of cell lines}

Lentiviral constructs expressing Arch-EGFP or EGFP (Control) were generated in the pLenti6.3/V5-DEST vector (Thermo Fisher Scientific K.K.). Viral transduction was performed as described previously ${ }^{13)}$. Lentiviral vectors and three packaging plasmids (pVSVG, pLG1, and pLG2) were transfected into 293FT cells using Lipofectamine 2000 (Thermo Fisher Scientific K.K.), and the culture medium (lentiviral solution) was collected $48 \mathrm{~h}$ later. Viral titers were estimated using Lenti-X quantitative reverse transcriptase polymerase chain reaction (PCR) (Chemicon, Temecula, CA, USA). Viral transduction of OBs was performed by adding the lentiviral solution to the culture medium in the presence of $6 \mu \mathrm{g} /$ $\mathrm{ml}$ polybrene. Stably transduced cells were selected with $5 \mu \mathrm{g} / \mathrm{ml}$ blasticidin.

\section{Electrophysiology}

Electrophysiological experiments were performed as described previously $^{10,14)}$. For whole-cell recordings, the standard external solution contained $145 \mathrm{mM} \mathrm{NaCl}, 5 \mathrm{mM} \mathrm{KCl}, 1 \mathrm{mM} \mathrm{CaCl}_{2}, 1 \mathrm{mM} \mathrm{MgCl}_{2}, 10$ $\mathrm{mM}$ glucose, $10 \mathrm{mM}$ HEPES (pH 7.3), and $0.1 \%$ bovine serum albumin. The pipette solution contained $130 \mathrm{mM} \mathrm{K}$-gluconate, $20 \mathrm{mM} \mathrm{KCl}, 3$ $\mathrm{mM} \mathrm{MgCl}{ }_{2}, 1 \mathrm{mM}$ EGTA, $1 \mathrm{mM}$ ATP, $0.5 \mathrm{mM} \mathrm{GTP}$, and $10 \mathrm{mM}$ HEPES ( $\mathrm{pH}$ 7.3). The $\mathrm{pH}$ of the bath and pipette solutions was adjusted using $\mathrm{NaOH}$ and $\mathrm{KOH}$, respectively. The osmolality of the solutions was maintained between 280 and $300 \mathrm{mOsm}$.

The borosilicate glass pipettes had a resistance of 5-8 $\mathrm{M} \Omega$. The reference electrode was an $\mathrm{Ag}-\mathrm{AgCl}$ wire connected to the bath solution through a Ringer-agar bridge. The zero-current potential before the formation of the gigaseal was taken as $0 \mathrm{mV}$. Current signals were recorded with an Axopatch 200A amplifier (Axon Instruments, Foster City, CA, USA), digitized at $1-2 \mathrm{kHz}$ with a Digidata 1200 analog-digital converter (Axon Instruments), and analyzed using pCLAMP software (Axon Instruments). Voltage steps from +60 to $-150 \mathrm{mV}$ were applied at a holding potential of $-30 \mathrm{mV}$ every $10-30 \mathrm{~s}$. The membrane potential was recorded in current-clamp mode.

\section{Light stimulus}

OBs stably expressing Arch-EGFP or EGFP only were seeded at 5.0 $\times 10^{5}$ cells $/ \mathrm{cm}^{2}$ in $\alpha$-MEM. Yellow-green light stimuli $(570 \pm 20 \mathrm{~nm}, 2-\mathrm{s}$ stimulus followed by a 1-s rest, repeated 10 times) was applied using an IX71 inverted microscope (Olympus, Tokyo, Japan) with a VMM-T1 fluorescence shutter controller (Uniblitz, Rochester, NY, USA). Light stimuli were applied every $24 \mathrm{~h}$ ( 1 time/day) or $12 \mathrm{~h}$ ( 2 times/day). To determine the contribution of Cav subtypes, the following specific Cav blockers were added during light stimulus: Diltiazem (Dil; L-type Cav blocker; Tocris Bioscience, Bristol, UK) or NNC 55-0396 (NNC: T-type Cav blocker; Alomone labs, Jerusalem, Israel). After the light stimulus, the culture medium was replaced to avoid the effects of Cav blockers.

\section{Gene expression assays}

Gene expression was measured as described previously ${ }^{5}$. Total RNA was extracted from the cultured cells using an RNeasy Kit (Qiagen KK, Tokyo, Japan) according to the manufacturer's protocol. First-strand cDNA was produced from total RNA using a High-Capacity cDNA Reverse Transcription Kit (Applied Biosystems, San Francisco, CA, USA). Quantitative real-time PCR was performed in a Step One Real-Time PCR System (Applied Biosystems) using SYBR Green and specific forward and reverse primers. Transcript levels were normalized to glyceraldehyde-3-phosphate dehydrogenase (GAPDH) transcript levels. The respective forward and reverse primer sequences were: ALP, 5'-gctatctctccettgcctgtatctg-3' and 5'-aggtgctttgggaatctgtgc-3';

Runt-related transcription factor 2 (Runx2), 5'-tggcttgggtttcaggttaggg-3' and 5'-tcggtttcttagggtcttggagtg- 3 '; Type I collagen, 5'-ctgactggaagagcggagag- $3^{\prime}$ and $5^{\prime}$-gcacagacggetgagtagg-3'; Osteocalcin $(\mathrm{OCN})$, $5^{\prime}$-gaatagactccggcgctacc-3' and 5'-agctcgtcacaattggggtt-3'; RANKL, 5'-gaaactcacagccetctctcttg-3' and 5'-gcatcggaatacctctcccaatc-3'; OPG, 5'-ggacgtcagctcttgtgtga-3' and 5'-gtagtcagggcaagggacac-3'; GAPDH, 5 '-agaaggtggtgaagcaggcat-3' and 5'-cgaaggtggaagagtgggagttg-3'.

\section{Statistical analysis}

Data are expressed as mean \pm standard error of the mean and were analyzed using the Student's $t$-test or analysis of variance. Tukey's honestly significant difference test was applied as a post-hoc test. The level of statistical significance was set at $p<0.05$. All analyses were performed using SPSS software (SPSS Japan, Tokyo, Japan).

\section{Results \\ Light-induced membrane hyperpolarization promotes osteoblast min- eralization.}

Our previous results showed that changes in membrane potential regulated intracellular RANKL transport ${ }^{10)}$. However, the effects of changes in membrane potential on osteoblast differentiation have not been investigated. First, we measured the membrane potential of OBs during osteoblast mineralization under whole-cell recording conditions (Fig. 1A). Five days after the induction of osteoblast mineralization, the membrane potential was hyperpolarized by culturing in osteogenic medium. Thus, we hypothesized that membrane hyperpolarization affects osteoblast differentiation. To allow non-invasive control of the membrane potential, we generated light-responsive OBs that stably expressed Arch, which is activated by yellow-green light and hyperpolarizes cells via proton transport ${ }^{7}$. Light-induced changes in membrane potential were recorded under the whole-cell clamp configuration in Arch-EGFP and control OBs. Arch-expressing cells were immediately hyperpolarized by $-16 \pm 1.2 \mathrm{mV}$ upon the onset of light stimulation and returned to the pre-stimulus potential after termination of the stimulus (Fig. 1B, C). The control cells did not respond to stimulation (Fig. 1D). Therefore, the temporal patterns of membrane potential could be strictly controlled with this light stimulus. This enabled us to investigate the effects of membrane hyperpolarization on osteoblast differentiation.

To examine the relationship between membrane hyperpolarization 
Takuya Notomi et al.: Effects of Membrane Hyperpolarization on Osteoblastic Mineralization
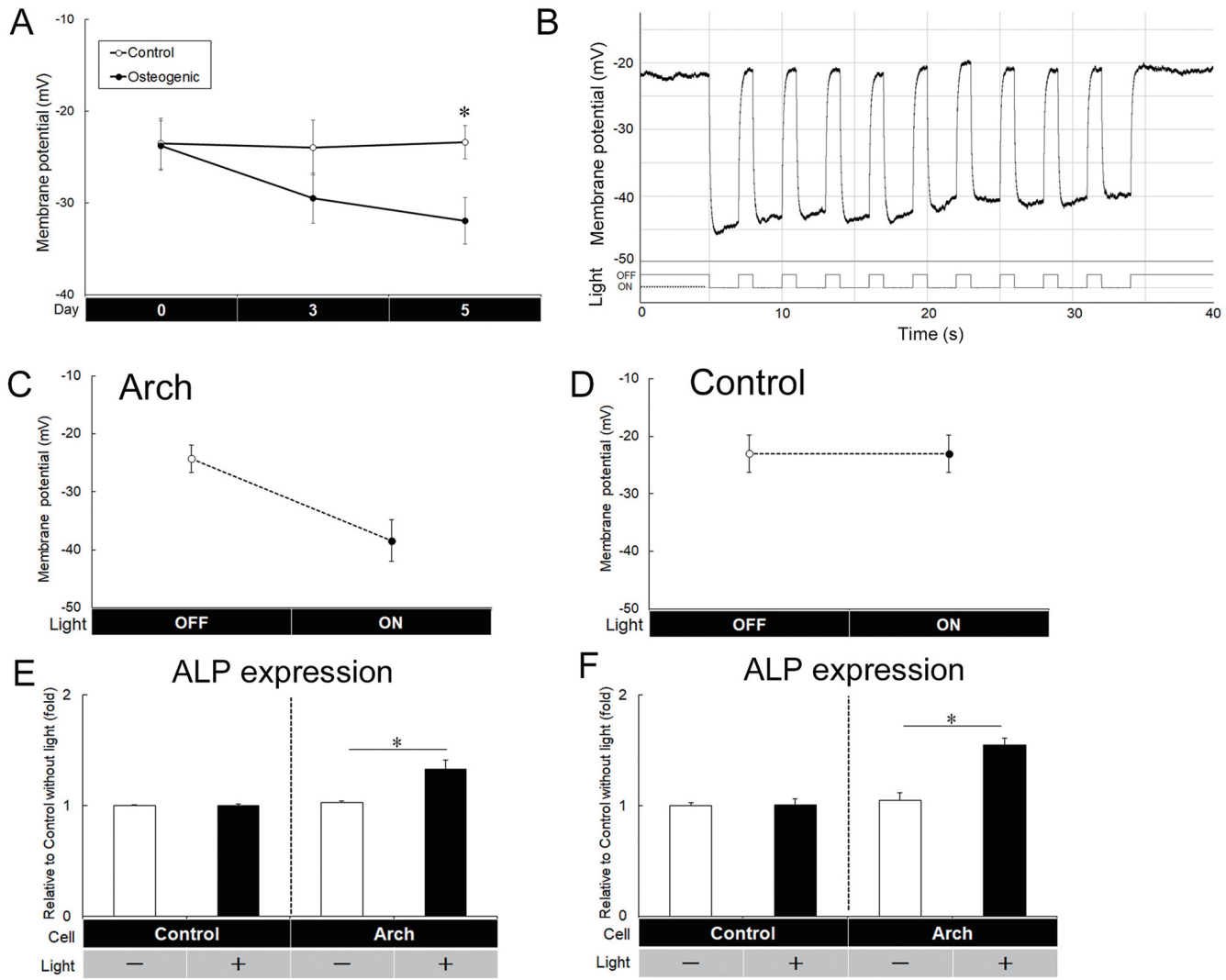

G

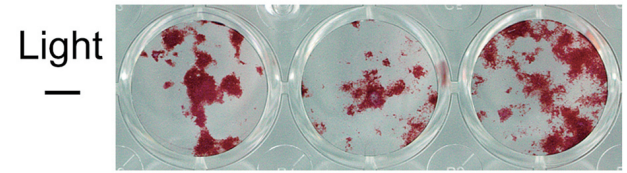

\section{$\mathrm{H}$}

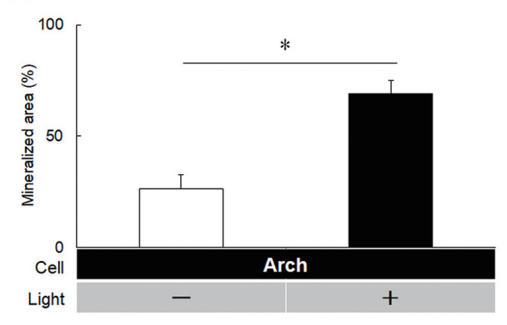

I

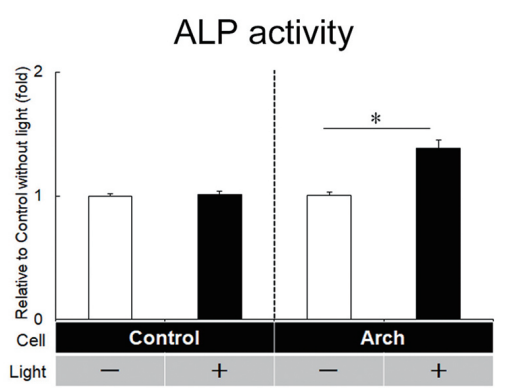

Figure 1. Light-induced membrane hyperpolarization promotes osteoblast differentiation in OBs. (A) Changes in membrane potential at 0,3 , and 5 days after the induction of mineralization. Control: cultured in alpha-MEM medium, Osteogenic: cultured in osteogenic medium. $(n=15-20)$. (B) Representative changes in the membrane potential induced by yellow-green light stimuli. Membrane hyperpolarization occurs when the light is turned on and terminated when the light is turned off. (C, D) Averaged hyperpolarization by light stimuli. Light stimuli hyperpolarized Arch-expressing cells (C) but not control (EGFP-expressing) cells (D). ( $\mathrm{n}=10-12)$. (E, F) Expression levels of alkaline phosphatase (ALP) in the absence or presence of light stimuli (E: 1 stimulus/day, F: 2 stimuli/day) in archaerhodopsin-3 (Arch) or control cells. Total RNA was extracted 7 days after the induction of mineralization. $(\mathrm{n}=5)$. $(\mathrm{G})$ Representative images of Alizarin Red staining for OB mineralization in the absence or presence of light stimuli. Staining was done 14 days after induction of mineralization. (H) The mineralized area based on Alizarin Red staining in the presence or absence of light stimuli for 14 days in Arch-expressed OBs. $(n=9)$. The mineralized area was normalized to the whole well area. (I) ALP activity in the absence or presence of light stimuli in Arch or Control cells, 12 days after the induction of mineralization $(n=6)$. Data are expressed as means $\pm \operatorname{SEM}(* p<0.05)$. 
and osteoblast differentiation, the conditions of the light stimuli were varied. A light stimulus (3 sets/stimulus; 1 set = "2 sec-ON, 1 sec-OFF" $\times 10$ times) was applied to Arch-expressing or control OBs at 1 stimulus/ day (every 24 h; Fig. 1E) or 2 stimuli/day (every 12 h; Fig. 1F). Seven days after induction of osteoblast differentiation, both conditions increased the expression levels of ALP, which is an early indicator of osteoblast mineralization (Fig. 1E, F). The condition of "1 stimulus/day" was chosen to reduce any artifacts of light exposure. At 14 days after induction of osteoblast mineralization, the light stimulus (1 stimulus/day) increased the alizarin red staining area in OBs expressing Arch-EGFP in comparison to the absence of light stimulus (Fig. 1G, H). ALP activity was measured (Fig. 1I). Light stimulus increased ALP activity in Arch, but not in Control. These data suggest that light-induced membrane hyperpolarization accelerates osteoblast mineralization. To further investigate the effects of membrane hyperpolarization on osteoblast mineralization, expression levels of early (Runx2 and Type I collagen) and late $(\mathrm{OCN})$ osteoblast differentiation markers were measured ${ }^{15,16)}$. Expression levels of Runx2, Type I collagen, and OCN were increased by light stimulus in Arch 7 days after induction of mineralization (Fig. 2A-C). These results confirmed that light-induced hyperpolarization promotes osteoblast mineralization. Next, we investigated the expression levels of osteoclastogenesis factors such as RANKL, OPG, and the RANKL/ OPG ratio (Fig. 2D-F). Similar to osteoblast differentiation markers, the expression levels of osteoclastogenesis factors were increased by light
A

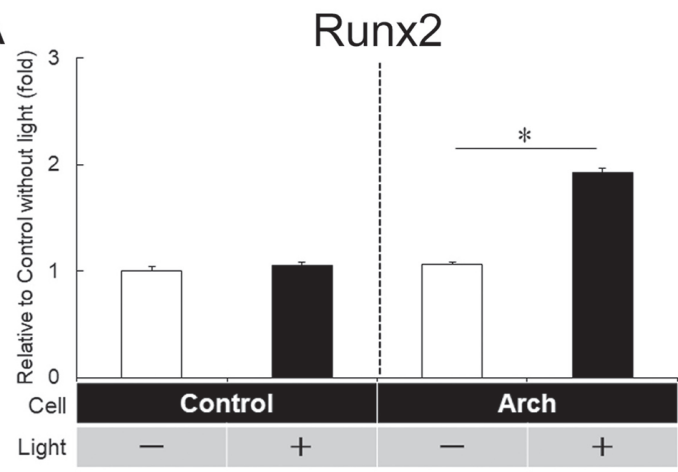

C

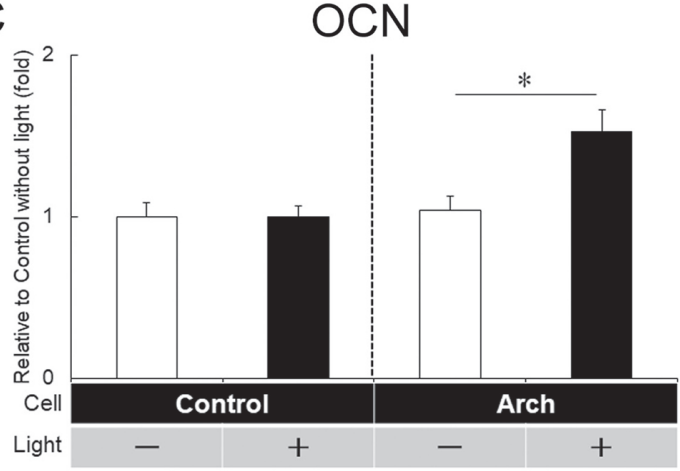

$\mathrm{E}$

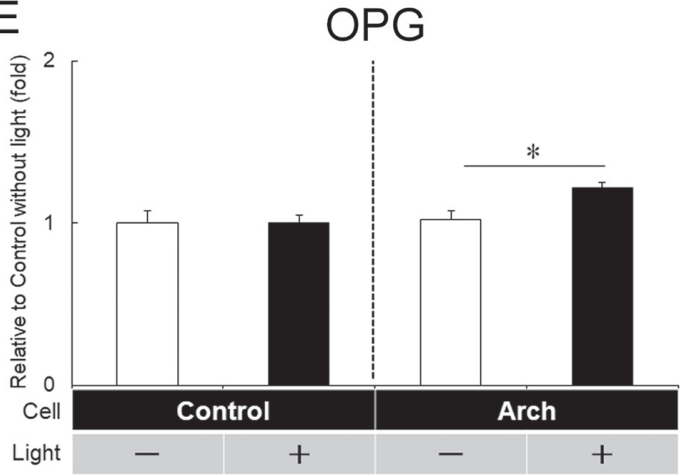

$\mathrm{B}$

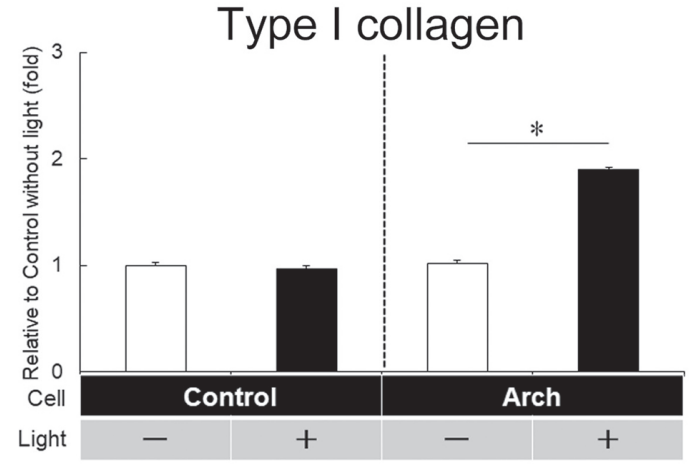

D

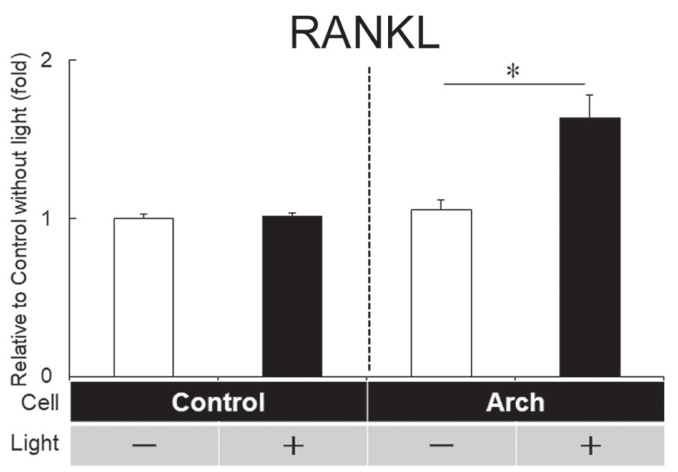

$\mathrm{F}$

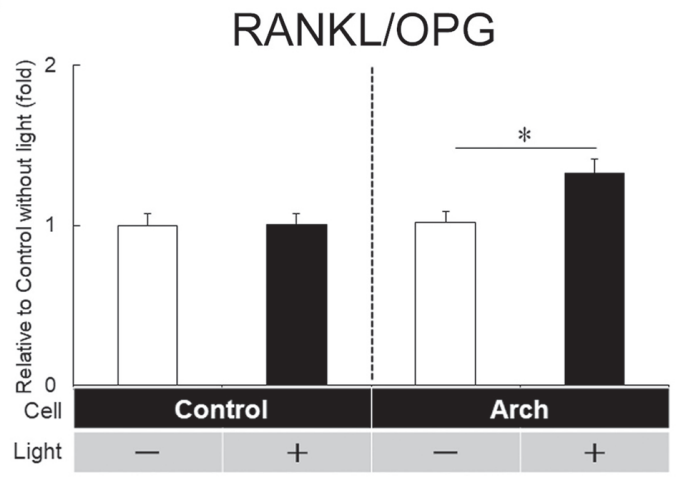

Figure 2. Expression of osteoblast mineralization markers and osteoclastogenesis factors are increased by light-induced membrane hyperpolarization. (A-C) Expression levels of osteoblast mineralization markers, Runx2 (A), Type I collagen (B), and OCN (C) in the absence or presence of light stimuli in Arch or Control cells. (D-F) Expression levels of osteoclastogenesis factors, RANKL (D), OPG (E), and RANKL/OPG ratio (F) in the absence or presence of light stimuli in Arch or Control cells. Total RNA was extracted 7 days after the induction of mineralization. Data are expressed as the mean $\pm \operatorname{SEM}\left(\mathrm{n}=5 ;{ }^{*} p<0.05\right)$. 
Takuya Notomi et al.: Effects of Membrane Hyperpolarization on Osteoblastic Mineralization
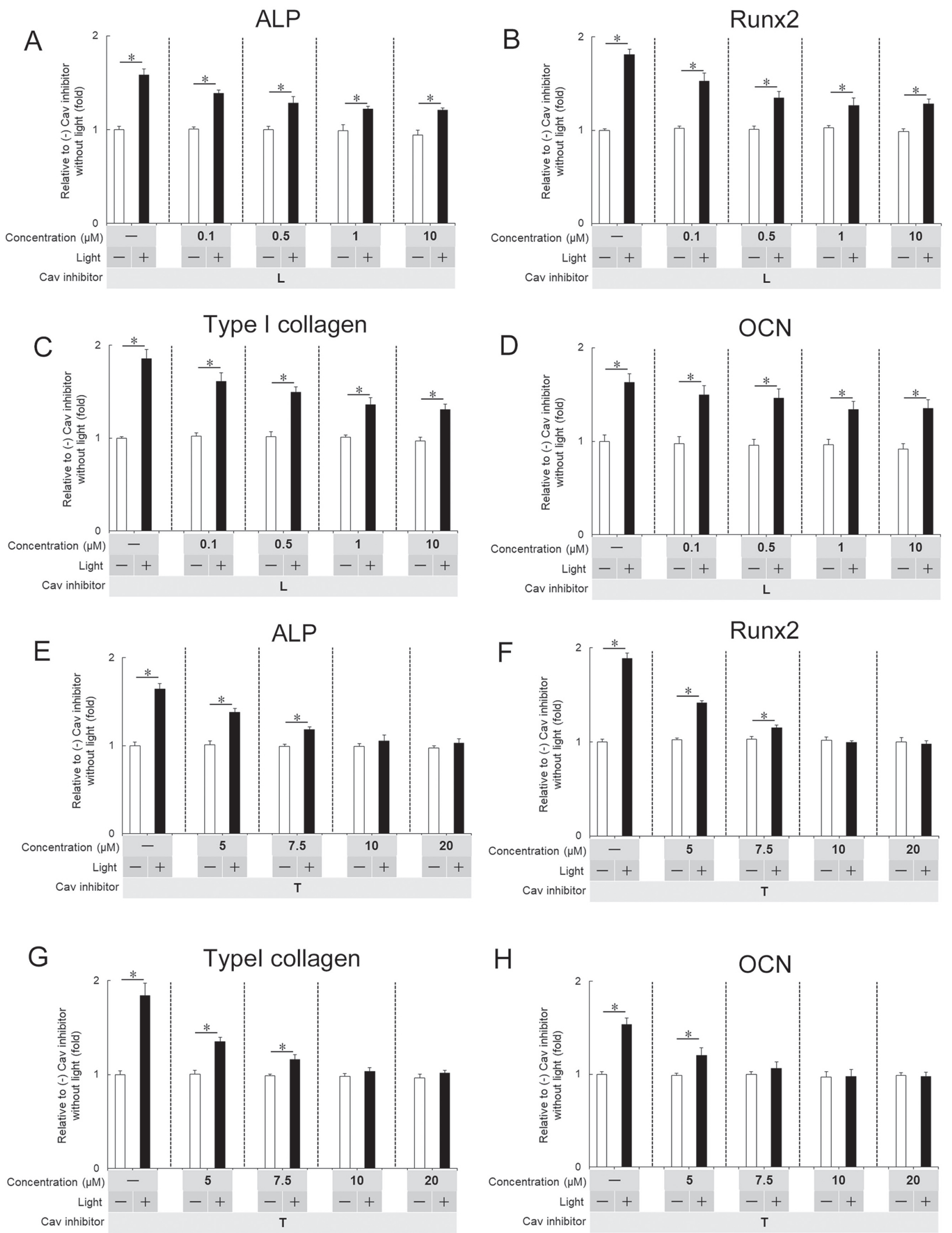

Figure 3. Cav inhibitors affect light-induced increment of expression levels of osteoblast mineralization markers. (A-D) Expression levels of osteoblast mineralization markers, ALP (A), Runx2 (B), Type I collagen (C), and OCN (D) in the absence or presence of light stimuli with different concentration of L-type Cav inhibitors in Arch cells. (E-H) Expression levels of osteoblast mineralization markers ALP (E), Runx2 (F), Type I collagen $(\mathrm{G})$, and OCN $(\mathrm{H})$ in the absence or presence of light stimuli with different concentrations of T-type Cav inhibitors in Arch cells. Total RNA was extracted 7 days after the induction of mineralization. Data are expressed as the mean \pm SEM $(\mathrm{n}=5 ; * p<0.05)$. 

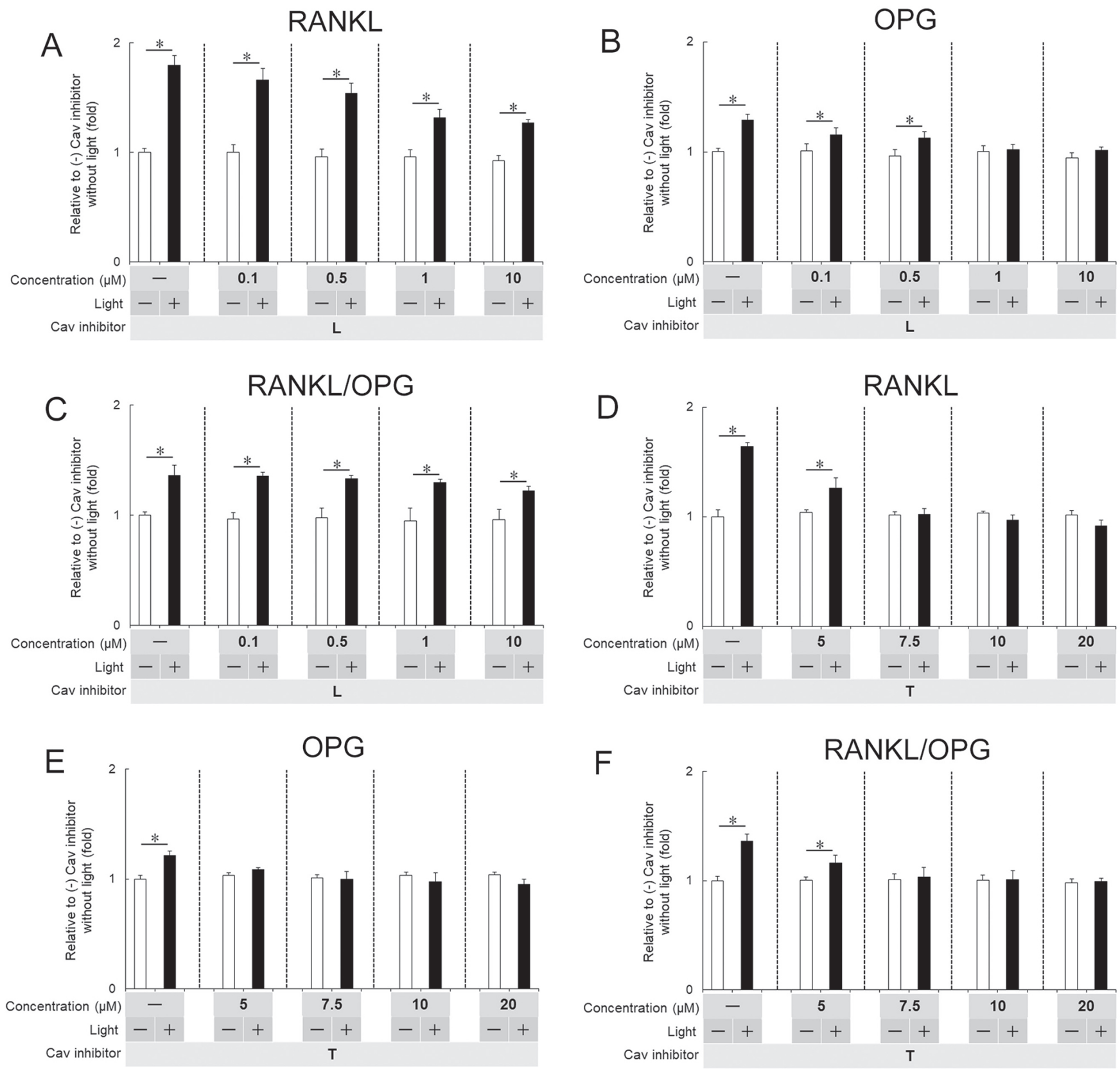

Figure 4. Cav inhibitors affect light-induced increment of expression levels of osteoclastogenesis factors in OBs. (A-C) Expression levels of RANKL (A), OPG (B), and RANKL/OPG ratio (C) in the absence or presence of light stimuli with different concentration of L-type Cav inhibitors in Arch cells. (D-F) Expression levels of RANKL (D), OPG (E), and RANKL/OPG ratio (F) in the absence or presence of light stimuli with different concentration of T-type Cav inhibitors in Arch cells. Total RNA was extracted 7 days after the induction of mineralization. Data are expressed as the mean $\pm \operatorname{SEM}(\mathrm{n}=5 ; * p<0.05)$.

stimulus in Arch but not in Control, suggesting that membrane hyperpolarization would affect osteoclastogenesis via RANKL/OPG signaling in OBs.

Cav inhibitors affect hyperpolarization-induced increment of osteoblast differentiation markers and osteoclastogenesis factors.

The changes in membrane potential would activate osteoblastic Cav. Previously we found that L-type (Cav1.2) and T-type (Cav 3.1 and Cav3.2) subunits were highly expressed. Thirty minutes before light stimulus, Diltiazem (Dil: a blocker for L-type Cav) or NNC55-0396
(NNC: a blocker for T-type Cav) were applied to the cells to block these Cav during light stimulus. Dil reduced the increase in osteoblast differentiation markers $\left(2^{\text {nd }}\right.$ vs. $4^{\text {th }}, 6^{\text {th }}, 8^{\text {th }}$ and $10^{\text {th }}$ column from the left; Fig. 3A-D); however, these expression levels were still significantly increased by light stimulus with a high concentration of Dil ( $9^{\text {th }}$ vs. $10^{\text {th }}$ column from the left; Fig. 3A-D). Inhibition of T-type Cav by addition of NNC reduced the increment of osteoblast differentiation markers $\left(2^{\text {nd }}\right.$ vs. $4^{\text {th }}, 6^{\text {th }}, 8^{\text {th }}$, and $10^{\text {th }}$ columns from the left; Fig. 3E-H). Furthermore, no significant differences were found in these markers with the addition of 10 and $20 \mu \mathrm{M} \mathrm{NNC} \mathrm{(} 7^{\text {th }}$ vs. $8^{\text {th }}$ and $9^{\text {th }}$ vs. $10^{\text {th }}$ column from the left; 
Fig. 3E-H). These findings indicate that the promotion of osteoblast differentiation by membrane hyperpolarization is mainly related to T-type $\mathrm{Ca}^{2+}$ channels.

In addition to osteoblast differentiation markers, we examined the expression levels of RANKL (Fig. 4A, D), OPG (Fig. 4B, E), and RANKL/OPG (Fig. 4C, F). $10 \mu \mathrm{M}$ Dil did not affect the light-induced increase in RANKL expression levels or the RANKL/OPG ratio $\left(9^{\text {th }} \mathrm{vs}\right.$. $10^{\text {th }}$ column from the left; Fig. 4A, C). However over $7.5 \mu \mathrm{M}$ NNC significantly inhibited the light-induced increase in RANKL, OPG, and RANKL/OPG (Fig. 4D-F). These results indicated that mainly T-type Cav affected the hyperpolarization-induced increment of osteoclastogenesis factor.

\section{Discussion}

The results of this study showed that light-induced membrane hyperpolarization accelerated osteoblast differentiation. Although controlling the membrane potential for long-term culture periods has been challenging, stable Arch-expressing OBs enable non-invasive control of the membrane potential. Light-induced membrane hyperpolarization increased the expression levels of osteoblast differentiation markers and osteoclastogenesis factors, and this increase was inhibited by Cav inhibitors. Because hyperpolarization of the membrane potential was observed during osteoblast differentiation, our results indicate that changing the membrane potential could be a novel approach for treating skeletal diseases.

Methods of optogenetics that regulate membrane potential by light stimuli have been reported ${ }^{7-9)}$. However, these methods have been applied mainly to excitable cells such as neurons, but not to non-excitable cells such as osteoblasts. We have reported that transient light stimulus-induced membrane depolarization facilitates the transport of intracellular vesicles within a few seconds ${ }^{10)}$. In this study, we showed that light-induced membrane hyperpolarization (1 stimulus/day) resulted in the promotion of osteoblast differentiation. Because the membrane potential during the mineralization process was hyperpolarized under whole-cell recording conditions, membrane hyperpolarization could be a key factor during osteoblast differentiation. It remains a hypothesis that, under physiological conditions, the osteoblastic membrane potential actively fluctuates, similar to the light-controlled membrane potential. Methods for non-invasive recording of membrane potential during osteoblast differentiation should be developed in future studies.

Our findings showed that membrane hyperpolarization increased the osteoblast differentiation markers and osteoclastogenesis factors, and that T-type Cav were mainly related to those increments, but the mechanism was not fully investigated. Some types of Cav have been reported to affect osteoblast mineralization ${ }^{3,4,6,17)}$, indicating that membrane hyperpolarization might activate these channels. Especially in T-type Cav, which are abundantly expressed in OBs and activated ${ }^{10)}$, membrane hyperpolarization could increase the open probability. Because T-type Cav are low voltage-activated $\mathrm{Ca}^{2+}$ channels, that become deinactivated during cell membrane hyperpolarization ${ }^{18)}$. To understand the relationship between changes in membrane potential and Cav throughout osteoblast mineralization, further long-term culture experiments are needed.

The present study adds to the novel role of membrane potential hyperpolarization in non-excitable cells. From the results of electrophysiological recordings, temporal patterns of osteoblastic membrane potential were controlled by a yellow-green light stimulus. Using our system, osteoblast mineralization was promoted by a single light stimulus per day in Arch-expressing cells. Our system accelerates the study of membrane potential in OBs and the search for novel drug candidates to treat bone disorders by increasing bone formation.

\section{Acknowledgments}

This work was supported in part by grants from the Japan Society for the Promotion of Science (JSPS) (JSPS KAKENHI: 20H05350, 18K09516, 21K09824). We would like to thank Editage (www.editage. com) for English language editing.

\section{Conflict of Interest}

All authors have no conflicts of interest.

\section{References}

1. Karsenty G and Wagner EF. Reaching a genetic and molecular understanding of skeletal development. Dev Cell 2: 389-406, 2002

2. Sims $\mathrm{N}$ and Martin TJ. Coupling the activities of bone formation and resorption: a multitude of signals within the basic multicellular unit. Bonekey Rep 3: 481, 2014

3. Gu Y, Preston MR, El-Haj AJ, Hamid J, Zamponi GW, Howl J and Publicover SJ. Osteoblasts derived from load-bearing bones of the rat express both L- and T-like voltage-operated calcium channels and mRNA for alpha $1 \mathrm{C}$, alpha $1 \mathrm{D}$ and alpha $1 \mathrm{G}$ subunits. Pflugers Arch 438: 553-560, 1999

4. Bergh JJ, Shao Y, Puente E, Duncan RL and Farach-Carson MC. Osteoblast $\mathrm{Ca}(2+)$ permeability and voltage-sensitive $\mathrm{Ca}(2+)$ channel expression is temporally regulated by 1,25-dihydroxyvitamin D(3). Am J Physiol Cell Physiol 290: C822-831, 2006

5. Suzuki T, Notomi T, Miyajima D, Mizoguchi F, Hayata T, Nakamoto T, Hanyu R, Kamolratanakul P, Mizuno A, Suzuki M, Ezura Y, Izumi $Y$ and Noda M. Osteoblastic differentiation enhances expression of TRPV4 that is required for calcium oscillation induced by mechanical force. Bone 54: 172-178, 2013

6. Wen L, Wang Y, Wang H, Kong L, Zhang L, Chen X and Ding Y. L-type calcium channels play a crucial role in the proliferation and osteogenic differentiation of bone marrow mesenchymal stem cells. Biochem Biophys Res Commun 424: 439-445, 2012

7. Chow BY, Han X, Dobry AS, Qian X, Chuong AS, Li M, Henninger MA, Belfort GM, Lin Y, Monahan PE and Boyden ES. High-performance genetically targetable optical neural silencing by light-driven proton pumps. Nature 463: 98-102, 2010

8. Nagel G, Szellas T, Huhn W, Kateriya S, Adeishvili N, Berthold P, Ollig D, Hegemann P and Bamberg E. Channelrhodopsin-2, a directly light-gated cation-selective membrane channel. Proc Natl Acad Sci USA 100: 13940-13945, 2003

9. Wang H, Sugiyama Y, Hikima T, Sugano E, Tomita H, Takahashi T, Ishizuka T and Yawo H. Molecular determinants differentiating photocurrent properties of two channelrhodopsins from chlamydomonas. J Biol Chem 284: 5685-5696, 2009

10. Notomi T, Kuno M, Hiyama A, Ezura Y, Honma M, Ishizuka T, Ohura K, Yawo H and Noda M. Membrane depolarization regulates intracellular RANKL transport in non-excitable osteoblasts. Bone 81: 306-314, 2015

11. Nifuji A, Ideno H, Ohyama Y, Takanabe R, Araki R, Abe M, Noda $\mathrm{M}$ and Shibuya H. Nemo-like kinase (NLK) expression in osteoblastic cells and suppression of osteoblastic differentiation. Exp Cell Res 316: 1127-1136, 2010

12. Ezura $Y$, Lin X, Hatta A, Izu Y and Noda M. Interleukin-1 $\beta$ Suppresses the transporter genes Ank and Ent1 expression in stromal progenitor cells retaining mineralization. Calcif Tissue Int 99: 199208, 2016 
13. Notomi T, Ezura Y and Noda M. Identification of two-pore channel 2 as a novel regulator of osteoclastogenesis. J Biol Chem 287: 35057-35064, 2012

14. Notomi T, Kuno M, Hiyama A, Nozaki T, Ohura K, Ezura Y and Noda M. Role of lysosomal channel protein TPC2 in osteoclast differentiation and bone remodeling under normal and low-magnesium conditions. J Biol Chem 292: 20998-21010, 2017

15. Stein GS and Lian JB. Molecular mechanisms mediating developmental and hormone-regulated expression of genes in osteoblasts. In: Cellular and Molecular Biology of Bone, ed by Noda M, Aca- demic Press, Inc., Tokyo, 1993, pp47-95.

16. Wei H, Shuying Y, Jianzhong S and Yi-Ping L. Signaling and transcriptional regulation in osteoblast commitment and differentiation. Front Biosci 12: 3068-3092, 2007

17. Li W, Duncan RL, Karin NJ and Farach-Carson MC. 1,25 (OH)2D3 enhances PTH-induced $\mathrm{Ca} 2+$ transients in preosteoblasts by activating L-type Ca2+ channels. Am J Physiol 273: E599-605, 1997

18. Dai S, Hall DD and Hell JW. Supramolecular assemblies and localized regulation of voltage-gated ion channels. Physiol Rev 89: 411452,2009 